\title{
INHERENT SIGNAL PREPROCESSING IN THE LINE CCD SENSOR
}

\author{
Luděk Kejzlar, Jan Fischer
}

\begin{abstract}
Czech Technical University, Faculty of Electrical Engineering, Department of Measurement;Technicka 2, 166 27, Prague 6, Czech Republic, kejzlal@feld.cvut.cz, fischer@feld.cvut.cz,

http://measure.feld.cvut.cz/usr/doctoral/kejzlar/index.html, http://measure.feld.cvut.cz/usr/staff/fischer/index.html
\end{abstract}

\begin{abstract}
This paper is devoted to the description and practical verification of a new line CCD sensor control method. This method is used for inherent signal preprocessing or processing in line CCD sensor. This new method is suitable for movement compensation, one-frame filtration or two-frame filtration. Because of the similarity between a NonRecursive Digital Filter with Finite Impulse Response (NRDF FIR) and the new mode we named it FIR mode. Conclusions and hints applicable for operation of line CCD sensor in FIR mode are also included in this paper.
\end{abstract}

Keywords: - CCD, Signal Processing, FIR Filter

\section{INTRODUCTION}

A FIR mode is one of the non-standard modes of a linear CCD sensor operation. We use this mode for inherent signal preprocessing in line CCD sensor. This mode is based on multiple exposures. A value of each output pixel is determined from values of $N_{F}$ previous photo-elements, which means $N_{F}$ exposures are needed to obtain an image. Each exposure has its own integration time, which has effect similar to the coefficients of filter impulse response.

\section{THE FIR MODE}

Output voltage in this mode is described by Eq. 1. Illuminance values of photo-elements $E_{n-j}$ are equal to samples of the image. Integration times $T_{X j}$ are equal to the coefficients of a FIR filter (impulse response). The entire frame integration time is equal to coefficient's sum. When we need to change the frame integration time all coefficients must by multiplied by ratio of old integration time and new integration time. Number of integration (exposures for one frame) $N_{F}$ is equal to the filter length. The $k_{r}$ and $k_{C}$ are conversion constants and $Q_{0}$ representing parasitic charges generated in the CCD sensor.

$$
u_{\text {OUT }}(i)=k_{r} \cdot k_{C} \cdot \sum_{j=0}^{N_{F}-1} E_{n-j} \cdot T_{X(j+1)}+N_{F} \cdot Q_{O}
$$

Fig. 1 shows a principle scheme of the line CCD sensor.

Sensor's signals and parts that we use in next text are shown in this figure.

\section{PRINCIPLE OF FIR MODE OF OPERATION}

FIR mode of operation can be build in simple control unit without complex arithmetic unit. All control functions are included in one simple subroutine. The subroutine flowchart is shown in Fig. 3. This subroutine

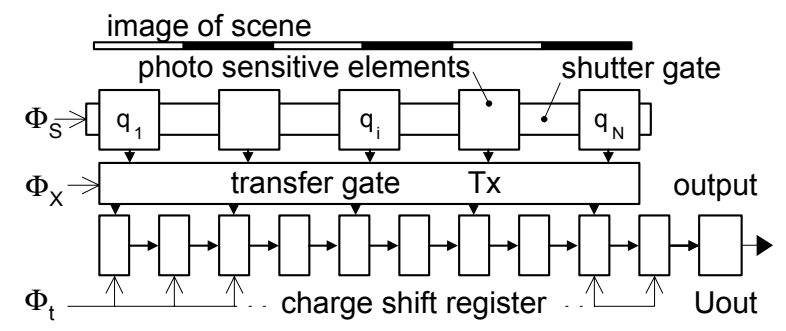

Fig. 1 - The line CCD sensor principle scheme.

corresponds to a cycle, which must be repeated $N_{F}$ times in order to obtain one frame. Here is described the Line CCD sensor control in FIR mode.

The sensor must be cleared at the beginning of each frame by electronic shutter (signal $\boldsymbol{\Phi}_{S}$ ). At the same time the first coefficient is written to the timer control register. In this case a timer is used for integration time generation. The control cycle is described below.

The charges are transported by control signal $\Phi_{X}$ to the charge shift register after a lapse of integration time (timer interrupt occurred). The transported charges are added to the charges stored there from the last cycle. If the last cycle (exposure) is executed the read-out is processed (the frame is complete) otherwise the charges in shift register are moved by 
one position by means of control signal $\Phi_{t}$.

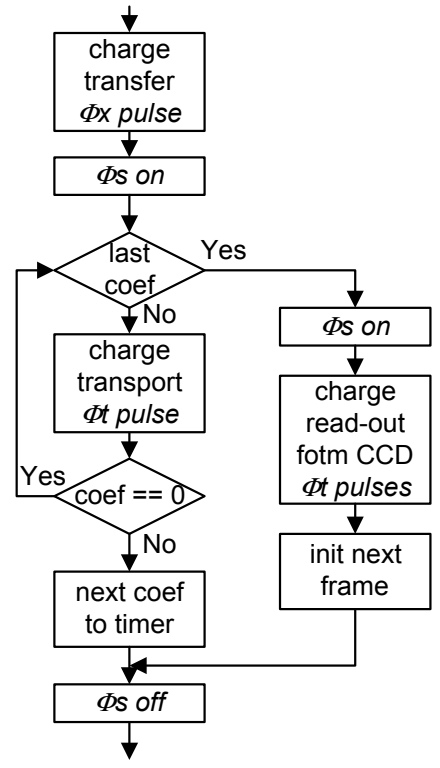

Fig. 2 - Flowchart of the FIR mode control algorithm.

The next coefficient is loaded to timer control register after the charge is moved. If the coefficient is equal to zero only next charge move is processed and the next coefficient is loaded. The next exposure begins after the timer is loaded.

\section{EXAMPLES OF FIR MODE APPLICATIONS}

There are many applications for the FIR mode of operation. Only two of them are discussed here, movement compensation and one-frame filtration (especially using one-frame filtration for data decimation).

Fig. 2 shows a block scheme of the line CCD camera we used for experimental verification of this mode.

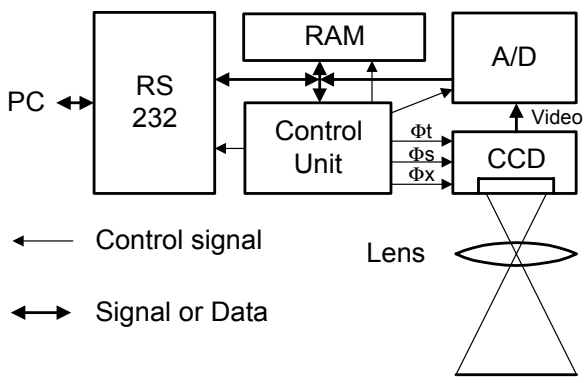

Fig. 3 - The line CCD camera principle scheme.

\section{MOVEMENT COMPENSATION}

An image of a moving object obtained with a long integration time is needed in some cases. It is impossible to obtain a sharp image using standard mode with long integration time because the object must not significantly change its position during the integration time. The measurement set-up for this reason is shown in fig. 4 .

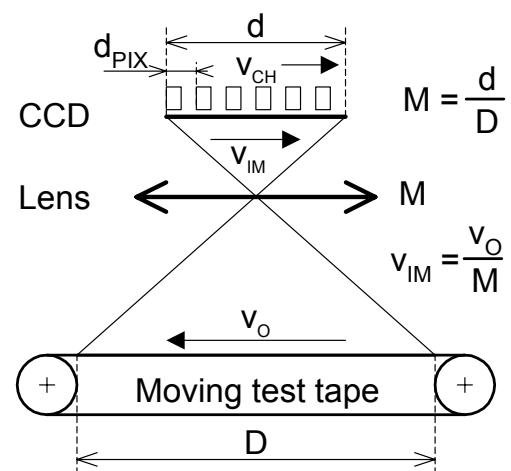

Fig. 4 - Measurement of moving object set-up.

The FIR mode of operation can be used even in this case. All FIR filter coefficients must have the same length in this case. The charge in the shift register has a velocity determined by coefficients length. The sharp image is obtained (coherence integration occurs) when the charge velocity in the shift register is equal to the velocity of the image on the CCD sensor. The frame has $N_{F}$ times higher illumination when it is obtained in FIR mode with $N_{F}$ coefficients then sharp frame obtained in standard mode. The optimum number of the coefficients depends on measurement conditions and may be calculated from eq. 2. The optimum integration time per one coefficient may be calculated as $t_{I N T}=T_{X} / N$.

$$
N=\left(v_{I M} \cdot T_{X}\right) \operatorname{div}\left(d_{P I X}\right)=\left(v_{O} \cdot T_{X}\right) \operatorname{div}\left(M \cdot d_{P I X}\right)
$$

where $T_{X}$ is required integration time and next conotation of the variables follows from the fig. 3 .

As an example are shown moving test tape image obtained using standard mode (in fig. 6) and image obtained by FIR mode with 32 coefficients (in fig. 7). Both images had same integration time $T_{X}$ equal to $9.344 \mathrm{~ms}$. Test tape velocity $v_{O}$ was 0.44 $\mathrm{m} / \mathrm{s}$. Charges velocity in charge shift register $v_{C H}$ was $0.048 \mathrm{~m} / \mathrm{s}$. Lens magnification was $M=0,11$. We can compare these images with reference static image obtained by standard mode (in fig. 5) obtained with the same conditions.

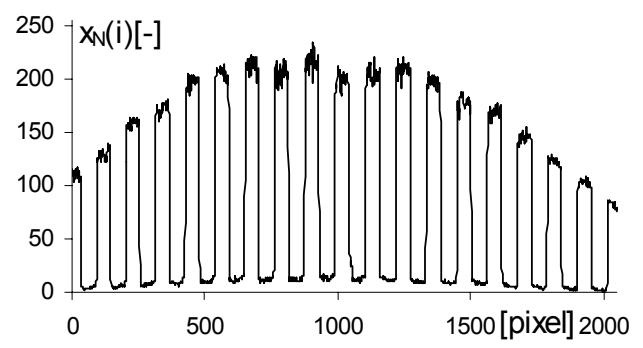

Fig.5 - Test tape static image obtained in Standard mode, The average edge width is 3 pixels. 


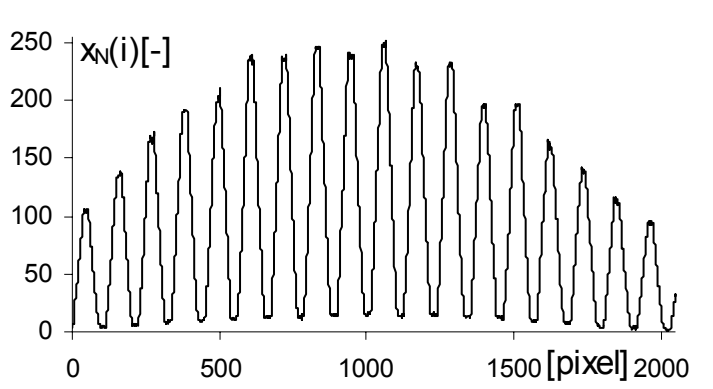

Fig.6 - Moving test tape image obtained in Standard mode. The average edge width is 23 pixels.

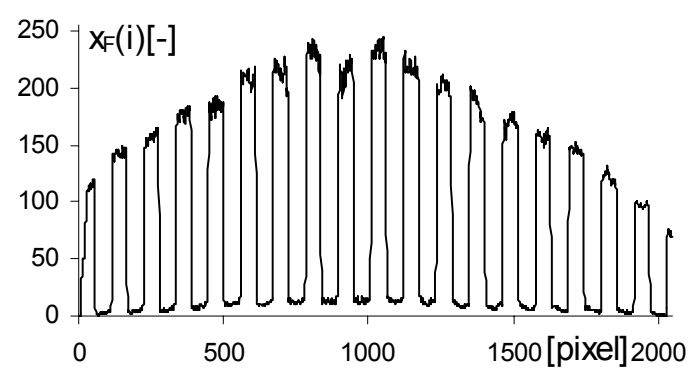

Fig.7 - Moving test tape image obtained in FIR mode. The average edge width is 5 pixels.

We can see that the image obtained in FIR mode has comparable widths of edges as the reference image. But image obtained in the standard mode has away wider edges.

\section{ONE-FRAME FILTRATION}

The one-frame filtration means that the FIR filter is calculated during obtaining one frame. We have a method called two-frame filtration where the complete filtration is done by subtracting two frames obtained with different coefficients. The one-frame filtration is calculated all in the line CCD sensor.

We used the one-frame filtration to build a low pass antialiasing filter for decimation. Decimation is correct way to decrease number of signal's samples by factor $K$. The relative frequencies higher then $1 / K$ must be removed from the signal before resampling by factor $K$ to avoid aliasing. For these purposes a low pass filter with relative stop frequency $1 / \mathrm{K}$ is used. Fig. 8 shows three ways of decimation realisation. Fig. 8a) shows decimation after A/D conversion, all video-signal pixles are converted. A digital low pas filter (DLP) is used. Fig. 8b) shows decimation before $A / D$ conversion, there is converted to digital only decimation number of pixels. An analogue LP filter (ALP) is used. Fig. 8c) shows the decimation we used. The LP filter is realised in the CCD sensor using FIR mode of operation and resampling is done before conversion.

This is a simple way to realise decimation. It is applicable for simple measuring systems such as a photoelectric gauge (sensor for measurement of dimensions).

a)

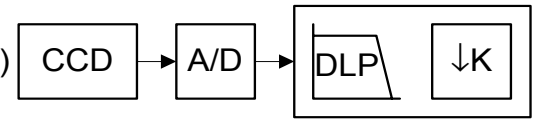

b)

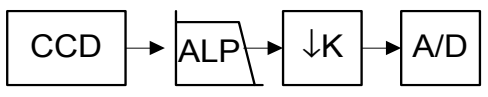

c)

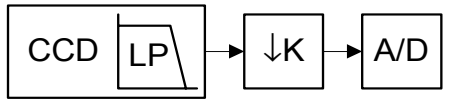

Fig. 8 - Decimation examples a) digital decimation scheme, b) decimation before $A / D$ conversion, $c)$ decimation scheme we used.

There is an example where the only resampling and correct decimation is used to decrease number of video-signal pixels. In this example we realised decimation with decimation factor $K$ equal to 8 . The low pass filter with relative stop frequency on $1 / K=$ 0,125 was designed for this decimation. The filter transfer function $|H(j \Theta)|$ of designed filter is shown in fig. 9.

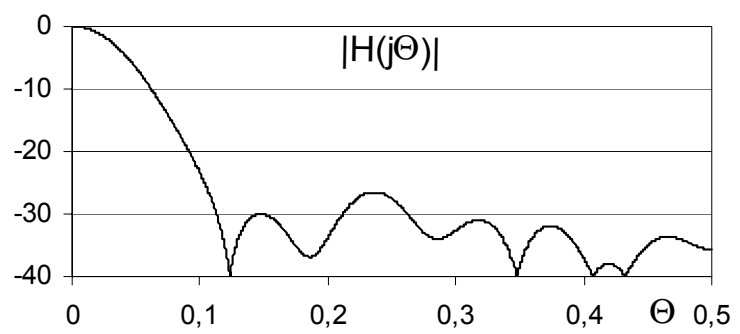

Fig. 9 - Transfer function of implemented LP filter.

Impulse response $h_{n}(i)$ of the filter is shown in fig. 10. Values of coefficients of the impulse response answer to FIR filter coefficients. The filter was implemented in CCD sensor as LP decimation filter.

A test with increasing spatial frequency was used for decimation verification. The full-scale test image is shown in fig. 11. This waveform is acquired by standard method of control CCD sensor.

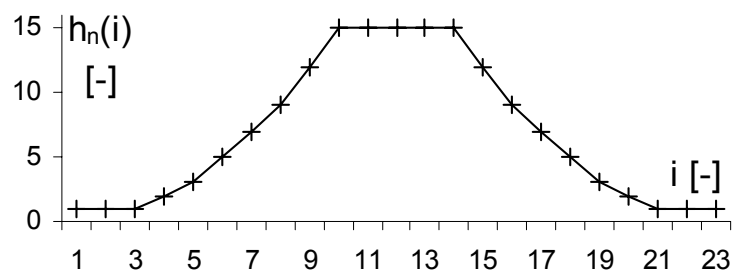

Fig. 10 - Impulse response of implemented LP filter.

Connection between relative frequencies of waveform (circles) and amplitude of the frequencies (line) from fig. 11 is shown in fig. 12. Both courses are shown in dependency to signal position. 


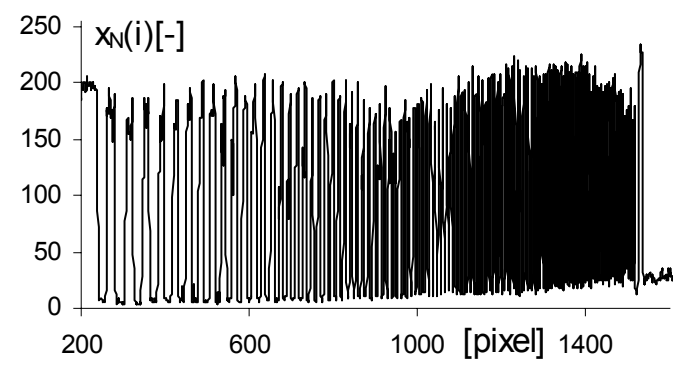

Fig. 11 - Image of the test obtained in normal mode.

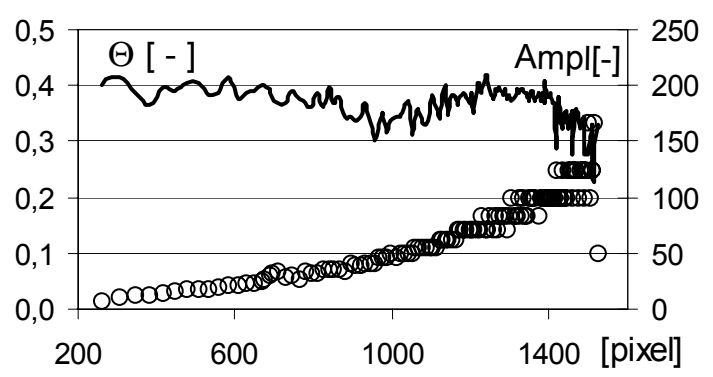

Fig. 12 - Representation of frequencies (circle) and its amplitude (line) in waveform from fig. 11.

Waveform of resampled signal from fig. 11 is shown in fig. 12. An aliasing occurred in this image.

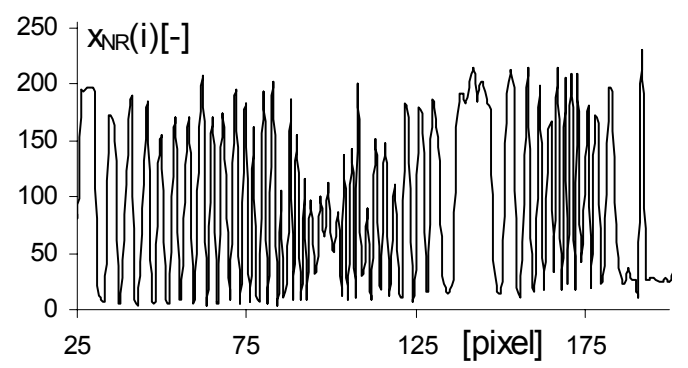

Fig. 13 - Test image resampled by factor $K=8$.

Connection between relative frequencies of waveform (circles) and amplitude of the frequencies (line) from fig. 13 is shown in fig. 14.

There you can see sliasing frequencies from $100^{\text {th }}$ pixel to $175^{\text {th }}$-pixel in fig. 14 (fig. 13).

The test image obtained by FIR mode (LP filter implemented) is shown in fig. 15 .

In fig. 16 is shown waveform of resampling signal from fig. 15. It is corresponds to correct decimation. There is no aliasing occurred.

In fig. 18 is shown the same situation as in fig. 14 for waveform acquired by FIR mode with LP filter and resampled with factor $\mathrm{K}=8$.

The ratio of power spectral density original signal to resampled signal is shown in fig. 18 resp. fig. 19. Here we can see that the aliasing occurs when only resampling is used. As the consequence of aliasing is occurrence of the false frequencies in image.

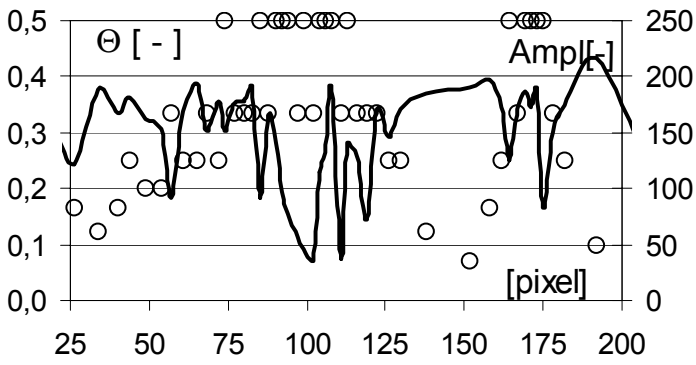

Fig. 14 - Representation of frequencies (circle) and its amplitude (line) in waveform from fig. 13.

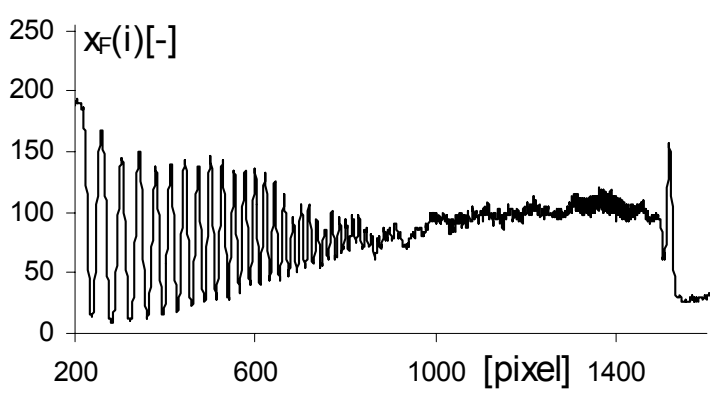

Fig. 15 - Waveform of test image obtained in FIR mode (LP antialiasing filter is implemented).

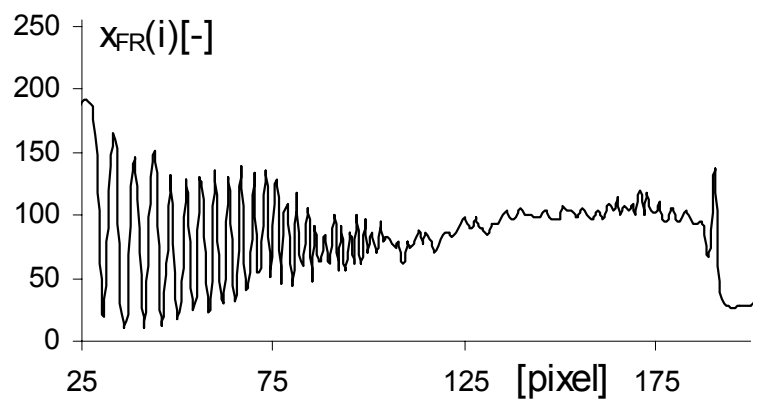

Fig. 16 - The resampled waveform from fig. 15 resampled by factor $K=8$.

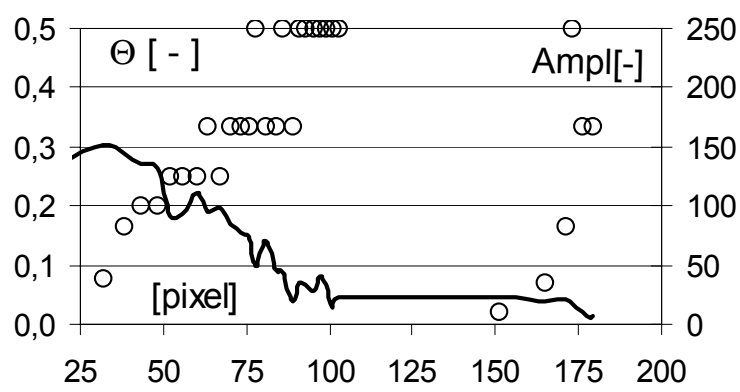

Fig. 17 - Representation of frequencies (circle) and its amplitude (line) in waveform from fig. 16.

But when the correct decimation with LP filter is used the aliasing does not occur.

This method is applicable for decreasing the pixel number in image. The method does not require complex control circuits. For example one chip 8 bit microcontrollers like AT89xx or AT90Sxx are applicable. 


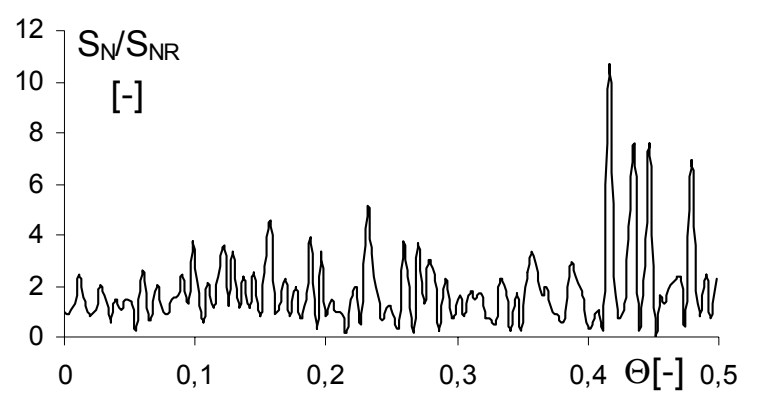

Fig. 18 - Power spectral density ratio of waveform acquired by standard mode to resampled waveform by factor $K=8$.

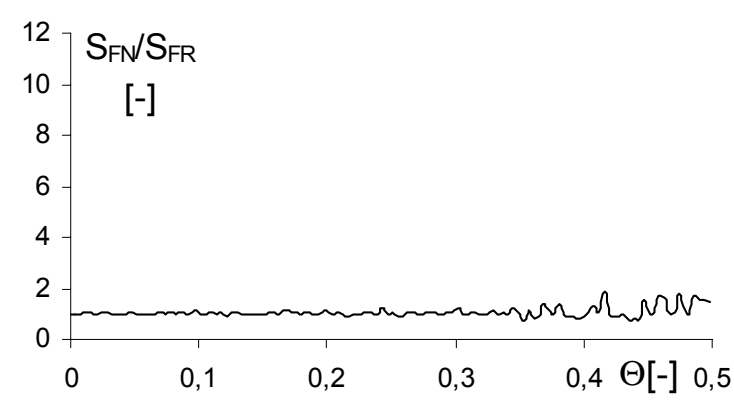

Fig. 19 - Power spectral density ratio of waveform acquired by FIR mode to resampled the waveform by factor $K=8$.

\section{CONCLUSIONS}

The FIR mode of line CCD sensor operation is usable for inherent signal pre-processing in line CCD sensor. It can by use for movement object scanning too. There are some advantages:

- the complex circuit for FIR filter calculation is not needed only the time interval control is necessary

- the calculation is processed without amplitude quantification

- the FIR filter calculation is executed during time of integration

But there is a problem with designing of positive impulse response FIR filter. This problem can be eliminated by two-frame filtration.

\section{ACKNOWLEDGEMENT}

This work has been supported by the research project MSM 210000015 „Research of New Methods for Physical Quantities Measurement and Their Application in Instrumentation".

\section{References}

[1] L. Kejzlar, J. Fischer. Signal Processing by Inherent FIR Filter in the Line CCD Sensor. pp. 215-218.

[2] F. Taylor. Principles of signals and systems. New York, 1994.

Luděk Kejzlar was born in Broumov, Czech Republic, on December 23, 1976. He received the Ing. (corresponding to the M.Sc. degree) in electrical engineering in 2001 at the Czech Technical University in Prague (CTU). At present time (2003) he is

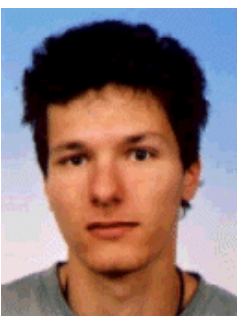
finishing the Ph.D. degree in electrical engineering at the CTU, Faculty of Electrical Engineering, Department of Measurement.

$\mathrm{He}$ is an instructor of seminars in CTU since 1998. He is working as a HW and SW developer in MapFactor s.r.o. since 2003. He mainly deal with measurement, especially contact-less measurement using the CCD sensors. He is focused on the nonstandard methods of control of CCD sensor.

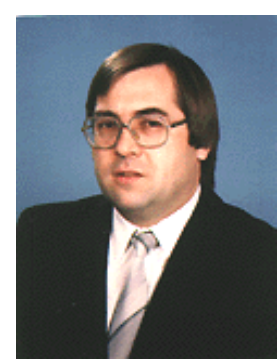

Jan Fischer was born in Kolin, Czech Republic, in 1953. $\mathrm{He}$ received the Czech equivalents of the M.Sc. and Ph.D. degrees in Electrical engineering from the Czech Technical University (CTU), Prague, in 1977 and 1989, respectively.

$\mathrm{He}$ is presently an assistant professor with Department of Measurement , Faculty of Electrical Engineering, CTU.

His pedagogical activities include lectures on Optoelectronic sensors, Videometry, Contactless measurement, Microprocessor techniques.

In his research work, he is concerned mainly with development of methods for image based measurement and application of CCD sensors and $D S P s r$ in the measuring instruments.

Since 1982 to 1992 he joined the company for production of automation systems ZPA - Kosire as Guest Researcher in Department for development of systems based on optoelectronic sensors. 